\title{
MicroRNA-302a enhances 5-fluorouracil-induced cell death in human colon cancer cells
}

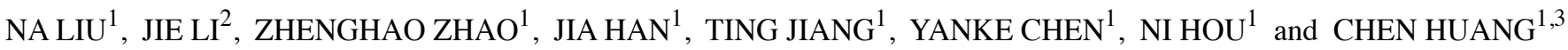 \\ ${ }^{1}$ Department of Cell Biology and Genetics, School of Basic Medical Sciences, ${ }^{2}$ Department of General Surgery, \\ The Second Affiliated Hospital, ${ }^{3}$ Key Laboratory of Environmentally and Genetically Associated Diseases, \\ Xi'an Jiaotong University Health Science Center, Xi'an, Shaanxi 710061, P.R. China
}

Received June 14, 2016; Accepted November 2, 2016

DOI: $10.3892 /$ or.2016.5237

\begin{abstract}
New therapeutic strategies are needed for colorectal cancer (CRC) treatment. MicroRNAs are involved in cancer-pertinent cellular processes, including chemoresistance. As miR-302a is an embryonic stem cell-specific microRNA, studies on miR-302a have focused on its role in human stem cells. Studies analyzing miR-302 function in cancer are limited. In this study, we used two human colon cancer cell lines, HCT116 and HT29, and evaluated the influence of miR-302a on 5-fluorouracil (5-FU)-induced cell death and viability inhibition. With bioinformatics tools, we hypothesized that insulin-like growth factor-1 receptor (IGF-1R) is a novel target of miR-302a, which we confirmed using a luciferase reporter assay and immunoblotting. Then, we designed siRNA against IGF-1R and found that si-IGF-1R resembled the effect of miR-302a on 5-FU treatment. Both miR-302a and si-IGF-1R inhibited Akt signaling. In conclusion, miR-302a targeted IGF-1R and enhanced 5-FU-induced cell death and viability inhibition in human colon cancer cells. Targeting miR-302a may offer new therapeutic interventions in CRC.
\end{abstract}

\section{Introduction}

Colorectal cancer (CRC) is the third most common cancer worldwide with an estimated 1.4 million cases and 693,900 deaths in 2012 (1). Its incidence in many developing countries has increased 2- to 4-fold over the past two decades (2). Surgical resection and adjuvant chemotherapy are the main CRC treatments. However, primary or acquired resis-

Correspondence to: $\mathrm{Dr} \mathrm{Ni}$ Hou or Professor Cheng Huang, Department of Cell Biology and Genetics, School of Basic Medical Sciences, Xi'an Jiaotong University Health Science Center, 76 Western Yanta Road, Xi'an, Shaanxi 710061, P.R. China

E-mail: houni@mail.xjtu.edu.cn

E-mail: hchen@mail.xjtu.edu.cn

Key words: microRNA-302a, insulin-like growth factor-1 receptor, 5-fluorouracil, Akt, human colon cancer cell tance is a major challenge to successful treatment (3). New therapeutic strategies and/or new adjuvant drugs are urgently needed.

5-Fluorouracil (5-FU), a cytostatic drug, is the most frequently used therapy and the backbone in CRC treatment. However, the response rates for 5-FU-based chemotherapy for advanced CRC are only $10-15 \%$. The combination of 5-FU with newer chemotherapies, such as irinotecan and oxaliplatin, improved the response rates to $40-59 \%$ (4). In the 5-FU metabolic pathway, four enzymes, including thymidylate synthase, methylenetetrahydrofolate reductase, dihydropyrimidine dehydrogenase and thymidine phosphorylase, are considered important to determining the sensitivity or resistance; however, data from many studies have been contradictory (5). Recently, amplified insulin-like growth factor-1 (IGF-1)/ IGF-1R signaling has been reported as critical for developing $\mathrm{CRC}$, and it contributes to CRC cell survival, invasion, metastasis and resistance to chemotherapeutic drugs by activating survival pathways, such as the phosphatidylinositol 3-kinase (PI3K)/Akt pathway (6-9).

Accumulating evidence suggests that microRNAs (miRNAs) are involved in many biological pathways and cancer-pertinent cellular processes, including cell proliferation, apoptosis, migration, invasion, stemness and chemoresistance $(10,11)$. Many studies have shown that miRNAs can modulate the sensitivity of cancer cells to anticancer drugs in substantial ways (12-15). miRNA-302a (miR-302a) belongs to the miR-302-367 cluster, which includes miR-302b, miR-302c, miR-302a, miR-302d and miR-367. This cluster was first identified in human embryonic stem cells (hESCs) and their malignant counterparts, human embryonic carcinoma cells (hECCs), and it has been reported to help maintain stemness and reprograming of somatic cells into induced pluripotent stem cells (iPSCs) by suppressing several key regulators of the G1/S transition, such as p21 and CCND $(16,17)$. In contrast, Lin et al reported that the subclass, miR-302a-d, inhibited tumorigenicity and induced apoptosis in various tumors and cancer cells, including MCF7 breast cancer cells, HepG2 hepatocellular carcinoma cells, and Tera- 2 embryonal teratocarcinoma cells by suppressing the CCNE-CDK2 and CCND-CDK4/6 pathways (18). Zhang et al also suggested that miR-302a inhibited prostate cancer cell proliferation by targeting Akt (19). Liu et al suggested that miR-302a sensitized 
testicular embryonal carcinoma cells to cisplatin-induced cell death in part through downregulating p21 (20). However, the roles of miR-302a in 5-FU treatment in CRC are unknown.

In this study, we used two human colon cancer-derived cell lines, HCT116 and HT29, and evaluated the influence of miR-302a on 5-FU-induced cell death and viability inhibition. With bioinformatics tools, we speculated that IGF-1R is a novel target of miR-302a, which was further confirmed using luciferase reporter assays and immunoblotting. Then, we designed siRNA against IGF-1R and observed that si-IGF-1R resembled the influence of miR-302a on 5-FU treatment. Thus, targeting miR-302a may offer new therapeutic interventions in $\mathrm{CRC}$.

\section{Materials and methods}

Materials. 5-FU was purchased from Sigma-Aldrich (St. Louis, MO). Anti-IGF-1R, anti-caspase-3, and anti-phosphorylated Akt antibodies were obtained from Cell Signaling Technology, Inc. (Beverly, MA, USA). Anti-cleaved PARP was purchased from Abcam (Cambridge, UK). Anti-Akt was from ProteinTech (Wuhan, China) and anti- $\beta$-actin was from Santa Cruz Biotechnology, Inc. (Dallas, TX, USA).

Cell culture. Human colon cancer cells (HCT116 and HT29) were a gift from Professor H. Kuwano (Gunma University, Gunma, Japan). The cells were cultured in RPMI-1640 medium (HyClone, Logan, UT, USA) supplemented with $10 \%$ fetal bovine serum (Biological Industries, Beit Haemek, Israel) at $37^{\circ} \mathrm{C}$ in a humidified atmosphere of $95 \%$ air and $5 \% \mathrm{CO}_{2}$ with medium change every 2 days. Cells in the mid-log phase were used in this study.

Patients and tissue samples. Surgically removed paired CRC tumor tissues and adjacent normal mucosal tissues were collected from 24 patients at the Second Affiliated Hospital of Xi'an Jiaotong University, and they were used for quantitative real-time polymerase chain reaction (qRT-PCR) analysis. Surgically resected tissues were quickly frozen in liquid nitrogen until analysis. All samples were obtained with the informed consent of the patients. This study was approved by the Medical Ethics Committee of the Xi'an Jiaotong University.

Plasmid construction and oligonucleotides. Human pre-miR-302a (MI0000738) (Table I) was chemically synthesized by Sangon Biotech (Shanghai, China) and cloned into the multiple cloning site (MCS) of pcDNA6.2-GW-miR vector (Invitrogen, Carlsbad, CA, USA), between EcoRI and HindIII, resulting in the pcDNA 6.2-pre-miR-302a construct (named by us). Empty pcDNA6.2-GW-miR vector was used as the control. pmirGLO dual-luciferase miRNA target expression vector (pmirGLO vector; Promega, Madison, WI, USA) was used to generate luciferase UTR reporter constructs. Specified fragments of the 3'UTR of human IGF-1R (Fig. 3A) were chemically synthesized and inserted into the MCS of the pmirGLO vector between SacI and XhoI, resulting in a plasmid that we named pmirGLO-IGF-1R-3'UTRWT. The mutant fragment of the 3'UTR of human IGF-1R (Fig. 3A) was also synthesized and inserted into the pmirGLO vector, resulting in a pmirGLO-IGF-1R-3'UTRMut plasmid. All subcloned frag- ments were directly sequenced. Small interfering RNA(siRNA) against IGF-1R (5'-CAAUGAGUACAACUACCGCUU-3') and the scrambled siRNA, as control, were obtained from Gene-Pharma Co. (Shanghai, China). Plasmids and siRNAs were transfected with Lipofectamine 2000 (Invitrogen) according to the manufacturer's instructions.

RNA isolation and $q R T-P C R$. Total cellular RNA was extracted using TRIzol (Invitrogen) and a miRNeasy mini kit (Qiagen) according to the manufacturer's instructions; miRNA levels were measured with qRT-PCR. Mature miRNAs were reverse transcribed into cDNA by stem-loop reverse transcription using the PrimeScript RT reagent kit (Takara, Otsu, Japan) and specific stem-loop primers, as shown in Table I; qRT-PCR was performed using FastStart Essential DNA Green Master on a light cycle 96 machine (both from Roche) according to the manufacturer's instructions. The relative expression of miRNA to internal control (U6 RNA) was calculated using the $2^{-\Delta \Delta C t}$ method. The primers used are listed in Table I.

Cell viability assay. Cell viability was determined by Trypan blue dye-exclusion assay. Cells were seeded at a concentration of $5 \times 10^{5}$ cells/well into a 6 -well plate. After treatment, they were collected and incubated with $0.4 \%$ Trypan blue dye. The stained and unstained cells were separately observed and counted on a hemocytometer under a light microscope (21). Cell viability was calculated according to the following formula: Cell viability $(\%)=$ (unstained cell number/total cell number for control) $x 100$. Cell death was calculated using the formula: Cell death $(\%)=($ stained cell number/total cell number) $\mathrm{x} 100$.

Cell apoptosis assay. Cell apoptosis was assessed using an Annexin V-FITC apoptosis detection kit (Invitrogen) according to the manufacturer's instructions. Cells were harvested and stained with Annexin V and fluorescein isothiocyanate. Then, cell apoptosis was examined using a flow cytometer (FACSCalibur), and histograms were analyzed using ModFit software (both from Becton-Dickinson, Franklin Lakes, NJ, USA).

Dual luciferase assay. PmirGLO-IGF-1R-3'UTRWT and pmirGLO-IGF-1R-3'UTRMut were, respectively, co-transfected with pcDNA 6.2-pre-miR-302a or pcDNA6.2 control vector into 293 cells in a 96-well plate using Lipofectamine 2000 (Invitrogen). At $48 \mathrm{~h}$ post-transfection, cells were lysed in $100 \mu \mathrm{l}$ of passive lysis buffer (Promega) and assayed according to the manufacturer's instructions. For each transfection, luciferase activity was averaged from five replicates. Luciferase activities were expressed as the ratio of Firefly to Renilla luciferase activity and normalized to the control level.

Western blot analysis. Cells were harvested from culture dishes and lysed in RIPA buffer supplemented with protease inhibitors and phosphatase inhibitors (Invitrogen). Protein concentrations were determined using a BCA protein assay kit (Pierce). Cell lysates (20 $\mu \mathrm{g}$ protein/lane) were separated by SDS-PAGE for PVDF membrane blotting. The blotted membranes were blocked with $5 \%$ skim milk for $30 \mathrm{~min}$ and incubated with primary antibodies (1:1,000 dilutions). The 
Table I. Primers and oligonucleotides used in this study.

\begin{tabular}{ll}
\hline Name & \\
\hline pre-miR-302a sense & PAimer sequences $\left(5^{\prime} \rightarrow 3^{\prime}\right)$ \\
& CATGTTTTGGTGATGGA \\
pre-miR-302a antisense & AGCTTCCATCACCAAAACATGGAAGCACTTACTTCTTTAGTTTCAAAGCAAGTACATC \\
& CACGTTTAAGTGGTGGG \\
miR-302a RT & GTCGTATCCAGTGCGTGTCGTGGAGTCGGCAATTGCACTGGATACGACTCACCAA \\
miR-302a F & ATCCAGTGCGTGTCGTGGA \\
miR-302a R & AGACGTAAGTGCTTCCATGTT \\
U6 RT & CGCTTCACGAATTTGCGTGTCAT \\
U6 F & GCTTCGGCAGCACATATACTAAAAT \\
U6 R & CGCTTCACGAATTTGCGTGTCAT \\
si-IGF-1R sense & CAAUGAGUACAACUACCGCUU \\
si-IGF-1R antisense & GCGGUAGUUGUACUCAUUGUU \\
si-Ctrl sense & UUCUCCGAACGUGUCACGUTT \\
si-Ctrl antisense & ACGUGACACGUUCGGAGAATT
\end{tabular}

$\mathrm{F}$, forward; R, reverse.

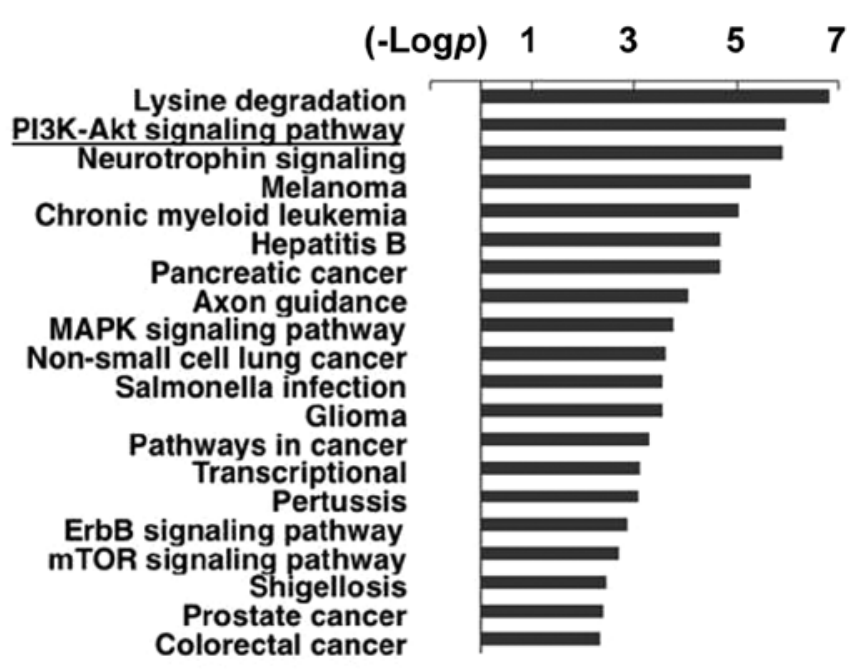

Figure 1. Bioinformatics pathway analysis based on the miR-302a target genes. DIANA-miRPath v2.0 was applied to analyze the main functions of miR-302a. The vertical axis is the KEGG pathway category, and the horizontal axis is the negative logarithm of the p-value (-Log p), which represents the significance of the pathways.

immunoreactive bands were visualized by enhanced chemiluminescence using horseradish peroxidase-conjugated IgG secondary antibodies. The band intensity was measured by densitometry and then quantified using Gel Plotting Macros of NIH image 1.62 and normalized to the indicated sample in the same membrane.

Bioinformative analysis. RegRNA (A regulatory RNA Motifs and Elements Finder http://regrna.mbc.nctu.edu.tw/) and TargetScan (http://www.targetscan.org/) were used for gene-related specified microRNA prediction. Through bioinformatics analysis, we obtained the predicted fragment of the targeted gene (IGF-1R), which was associated with miR-302a. DIANA-miRPath v2.0 (http://www.microrna.gr/miRPathv2) was applied to analyze the main functions of miR-302a (22). The one-tailed Fisher's exact test was used to identify the enriched KEGG pathways with targets of miR-302a, and the false discovery rate (FDR) was calculated to correct the p-value. Enrichment provides a measure of the significance of the function; as the enrichment increases, the corresponding function is more significant.

Statistical analysis. Experiments were independently performed with three replicates, and data were expressed as the means \pm the standard deviation (SD). Statistical analysis was performed using Student's t-test, and $\mathrm{P}<0.05$ was considered statistically significant.

\section{Results}

miR-302a enhances 5-FU-induced viability inhibition in HT29 and HCT116 cells. Using bioinformatics software, we found that the function of miR-302a is significantly related to the PI3K-Akt pathway (Fig. 1), which is closely associated with cell survival and 5-FU resistance (23). To explore the role of miR-302a in 5-FU-induced cytotoxicity, we constructed a pre-miR-302a expression plasmid and transfected it or the control vector (miR-Ctrl) into HT29 and HCT116 cells. The qRT-PCR results showed that a sizable increase in miR-302a was achieved in both cells (Fig. 2A and B). This increase may be due to the very low expression of miR-302a in typical HT29 and HCT116 cells such that small changes in the miR-302a expression could be discerned after transfection of miR-302a inhibitor (data not shown). Based on previous data $(24,25)$, we used 25 and $10 \mu \mathrm{M}$ of 5-FU in HT29 and HCT116, respectively, and observed changes at $48 \mathrm{~h}$ of 5-FU treatment. As the data show, although $48 \mathrm{~h}$ of miR-302a overexpression had 
A

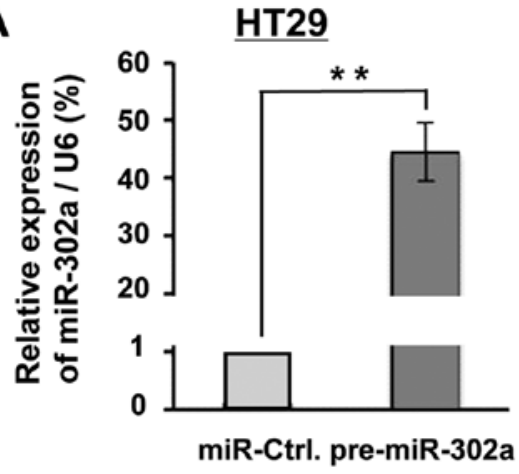

C

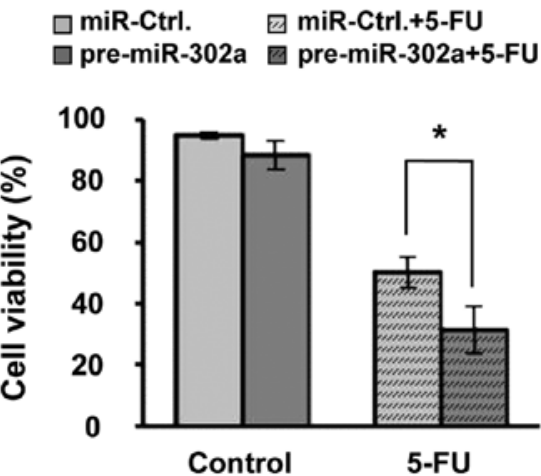

B

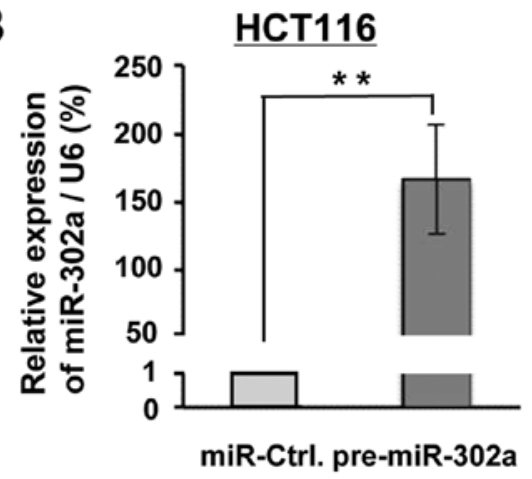

D
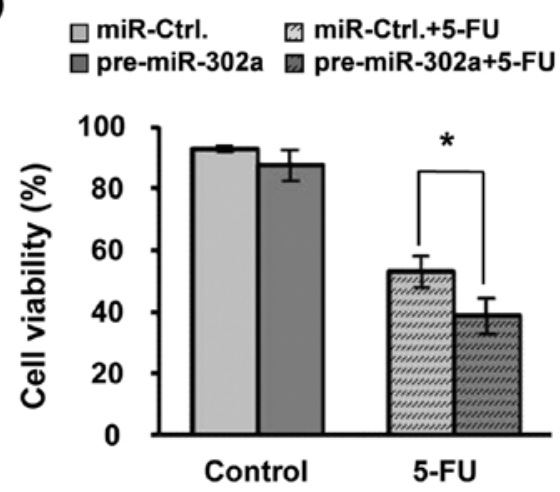

Figure 2. Overexpression of miR-302a enhances 5-FU-induced inhibition of cell viability in HT29 and HCT116 cells. HT29 and HCT116 cells were transfected with pcDNA6.2-pre-miR-302a or pcDNA6.2 vector (miR-Ctrl). The miR-302a level in (A) HT29 or (B) HCT116 cells was measured by qRT-PCR using U6 RNA as an endogenous control. Next, cells were transfected as before, and $6 \mathrm{~h}$ later, they were treated with 5-FU (HT29, $25 \mu \mathrm{M} ; \mathrm{HCT} 116,10 \mu \mathrm{M})$ or not; then, $48 \mathrm{~h}$ later, the cell viability was measured with the Trypan blue dye-exclusion assay [(C) HT29; (D) HCT116]. Experiments were repeated three times independently with reproducible results. ${ }^{*} \mathrm{p}<0.05$ and ${ }^{* *} \mathrm{p}<0.01$.

not significantly decreased the cellular viability, it enhanced the 5-FU-induced viability inhibition, from 50.22 to $31.44 \%$, in HT29 cells (Fig. 2C) and from 53.00 to $38.69 \%$ in HCT116 cells (Fig. 2D). These results suggested that miR-302a could enhance the 5-FU-induced viability inhibition in human colon cancer cells.

miR-302a increases 5-FU-induced cell death. The 5-FU-induced cell death, including apoptosis, is also a mechanism of 5-FU action (4). We measured cell death in our experiment. As with changes in viability, miR-302a overexpression did not significantly influence cell death in the cell lines; however, the combination of miR-302a with 5-FU remarkably increased cell death with $50.00 \%$ increase in HT29 and $62.72 \%$ increase in HCT116 (Fig. 3A and B). Apoptosis induced by 5-FU was also increased by the combination (Fig. 3C and D). We further detected apoptotic proteins. The data showed that 5-FU affected caspase-3 and its substrate, activated PARP; miR-302a further enhanced 5-FU activation (Fig. 3E and F). These results indicated that miR-302a could increase 5-FU-induced cell death, including apoptosis, in human colon cancer cells.

$m i R-302$ a targets IGF-1R. To search for the target gene of miR-302a involved in 5-FU action, we utilized RegRNA 2.0 and TargetScan bioinformative tools and found that in the 3'UTR of the IGF-1R gene at 5404-5437 nt, there is a potential miR-302a binding site (Fig. 4A). We then subcloned this fragment into the luciferase pmirGLO vector. We also mutated the binding site and inserted it into the pmirGLO vector. A dual luciferase assay was subsequently performed. We observed a significant decrease in the luciferase activity of IGF-1R WT in the presence of miR-302a compared with miR-Ctrl (Fig. 4B). However, no change in the IGF-1R Mut luciferase activity was detected between miR-302a and miR-Ctrl. Furthermore, miR-302a targeting to IGF-1R was confirmed in HT29 and HCT116 cells. Western blot analysis showed that ectopic miR-302a downregulated IGF-1R expression (Fig. 4C). Beyond the data in the luciferase assay, targeting of miR-302a to IGF-1R is also echoed by its downregulation (Fig. 4D) and the amplified IGF-1R signaling (7) in human CRC tissues. These data indicate that IGF-1R is a direct target of miR-302a.

Repression of IGF-1R enhanced 5-FU-induced viability inhibition and cell death. Next, we designed and synthesized si-IGF-1R and explored whether repression of IGF-1R could influence 5-FU treatment, such as with miR-302a. The IGF-1R expression was indeed repressed by the transfection of si-IGF-1R compared with si-Ctrl in HT29 (Fig. 5A) and HCT116 (Fig. 5B) cells. Additionally, the inhibition of cell viability by $5-\mathrm{FU}$ was enhanced by si-IGF-1R transfection, from 51.21 to $37.94 \%$ in HT29 (Fig. 5C) and from 53.60 to $32.11 \%$ in HCT116 (Fig. 5D) cells, although there were no significant changes by si-IGF-1R transfection alone. Changes in cell death were also measured. In both cell types, IGF-1R repression enhanced 5-FU-induced cell death; there was a $42.35 \%$ increase in HT29 and $54.78 \%$ 
A

HT29

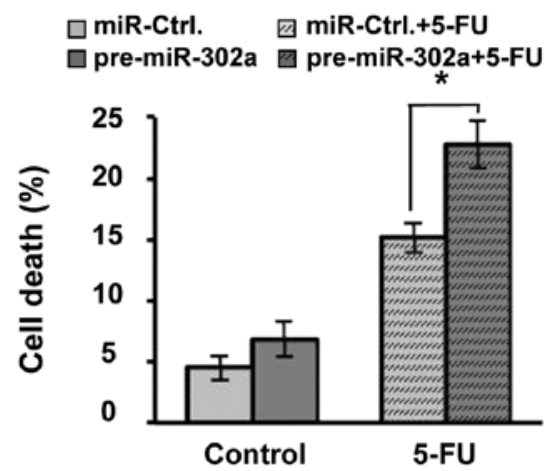

C
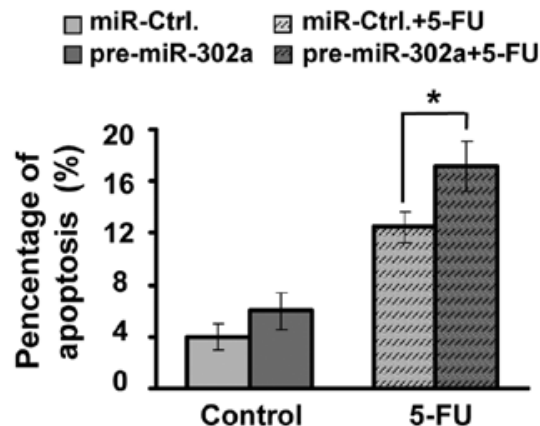

E

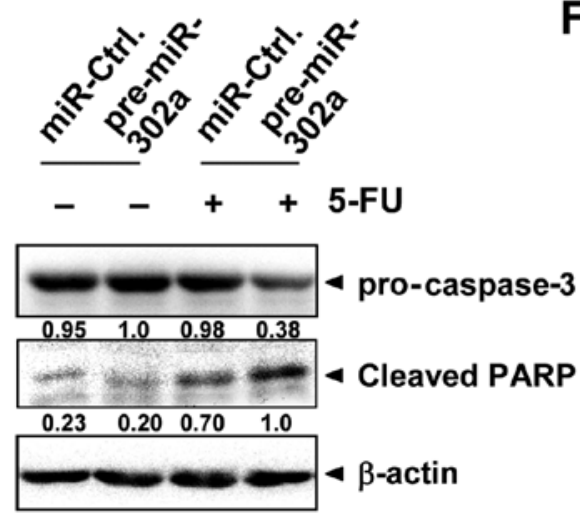

$\mathbf{F}$
B

HCT116

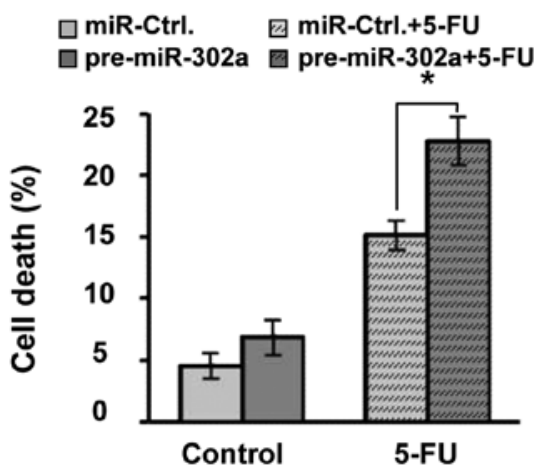

D
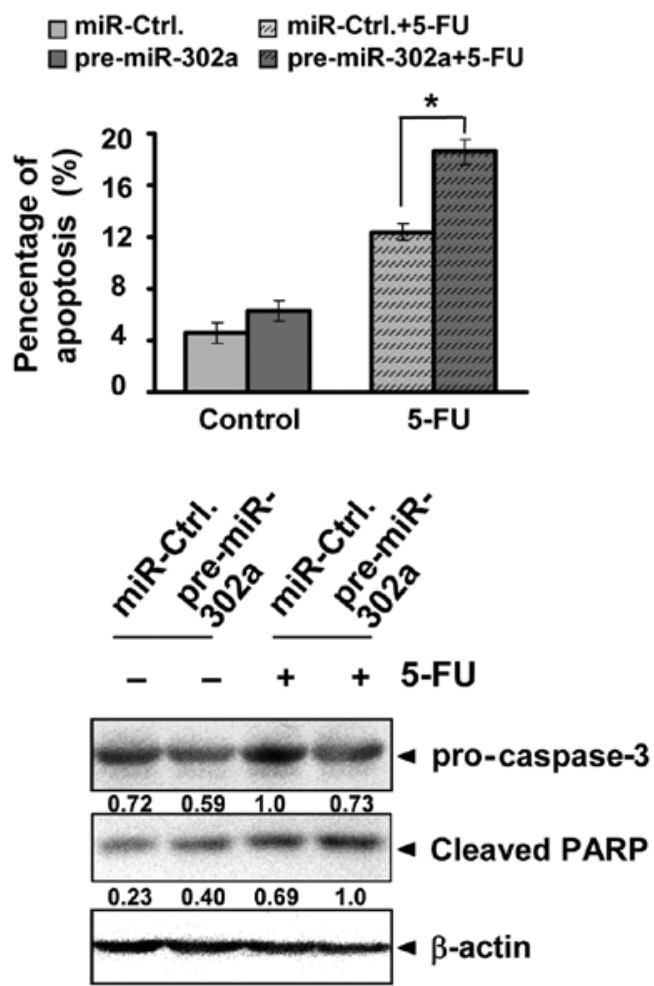

Figure 3. Overexpression of miR-302a increases 5-FU-induced cell death. HT29 and HCT116 cells were treated as in Fig. 2. Cell death was determined by the Trypan blue dye-exclusion assay [(A) HT29; (B) HCT116]. Cell apoptosis was measured using an Annexin V-FITC apoptosis detection kit [(C) HT29; (D) HCT116]. Furthermore, cell lysates were harvested, and the same protein level was loaded onto SDS-PAGE gels. (E,F) The pro-caspase-3 and cleaved PARP levels were detected using western blot analysis. $\beta$-actin was used as the loading control. Data are representative of three independent experiments. ${ }^{*}<0.05$.

increase in HCT116 (Fig. 6A and B). Apoptosis induced by 5-FU was also increased by the combination (Fig. 6C and D). Apoptotic proteins supported these changes. Caspase 3 and its substrate, PARP, were more activated by the combination of 5-FU and si-IGF-1R than by 5-FU alone in HT29 (Fig. 6E) and HCT116 (Fig. 6F) cells. These results suggested that IGF-1R repression could enhance 5-FU-induced viability inhibition and cell death in human colon cancer cells, resembling the influence of miR-302a on 5-FU treatment.

miR-302a inhibits Akt signaling. IGF-1R plays an important role in mediating the $\mathrm{PI} 3 \mathrm{~K} / \mathrm{Akt}$ pathway activation. We then examined whether miR-302a-triggered IGF-1R suppression was reflected in regulation of PI3K/Akt signaling in colon cancer cells (Fig. 7). IGF-1R repression inhibited constitutive Akt activation, as determined by the expression of phosphorylated Akt in HT29 and HCT116 cells. Akt is reportedly activated by $5-\mathrm{FU}$ and plays an important role in 5-FU chemoresistance $(23,26)$. In our experiment, 5 -FU treatment enhanced Akt activation, while the combination of 5-FU and IGF-1R repression inhibited Akt activation, which was consistent with changes in the cell and cell viability results. As expected, miR-302a overexpression also caused inhibition of Akt activation, and miR-302a combined with 5-FU significantly inhibited 5-FU-induced Akt activation. In addition, miR-302a decreased the expression of Akt, which was in agreement with a previous study (19). These results suggested that miR-302a could target IGF-1R and Akt as well as inhibit Akt signaling, and miR-302a overexpression could influence the 5-FU-induced cell death and viability. 
A

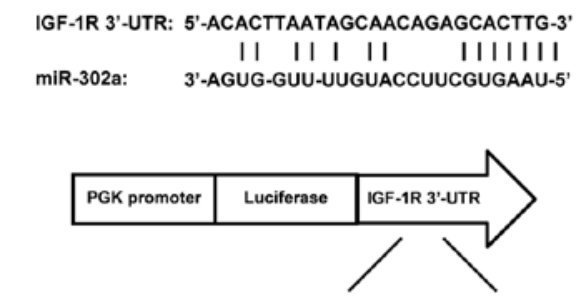

PGLO-IGF-1R 3'UTR WT: 5'-ACACTTAATAGCAACAGAGCACTTG-3' PGLO-IGF-1R 3'UTR MUt: 5'-ACACTTAATAGCAACAGAGTGTTTG-3'

C

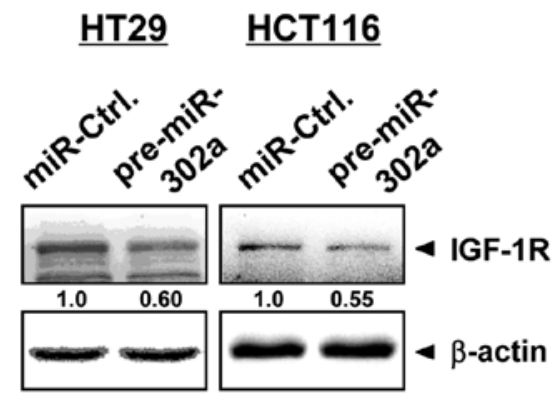

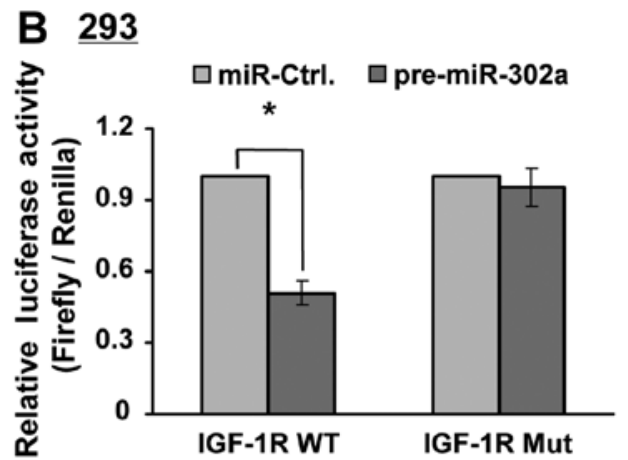

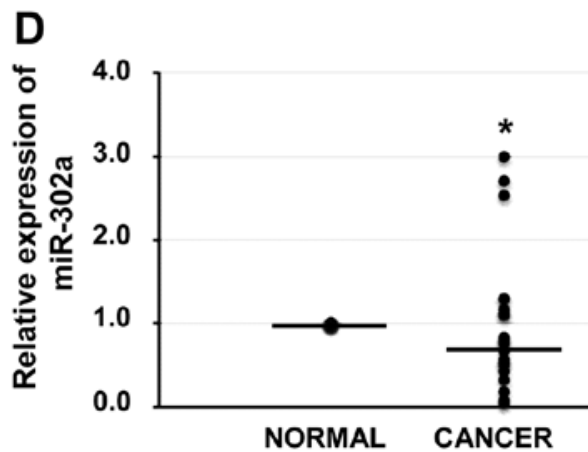

Figure 4. miR-302a directly targets IGF-1R by binding to the 3'UTR. (A) A putative miR-302a-binding site exists in the 3'UTR of IGF-1R and three point mutations were generated in the seed region (also the binding site) as indicated by underlining. The target sequence and its mutated sequence were subcloned into the pmirGLO vector, resulting in two plasmids, pGLO-IGF-1R-3'UTRWT and pGLO-IGF-1R-3'UTRMut. (B) These plasmids were respectively co-transfected with pcDNA 6.2-pre-miR-302a or pcDNA6.2 vector (miR-Ctrl) into 293 cells. At $48 \mathrm{~h}$ post-transfection, the dual luciferase assay was performed and the luciferase activities were expressed as the ratio of Firefly to Renilla luciferase activity and normalized to the level of control (miR-Ctrl). (C) Ectopic expression of miR-302a in HT29 and HCT116 cells decreased the IGF-1R protein levels according to western blot analysis. (D) Expression of miR-302a in 20 pairs of CRC tumor tissues and adjacent normal tissues was measured by qRT-PCR using U6 RNA as an endogenous control. Data are representative of three independent experiments. ${ }^{*} \mathrm{p}<0.05$.

A

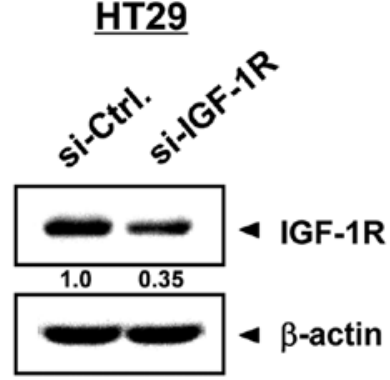

C
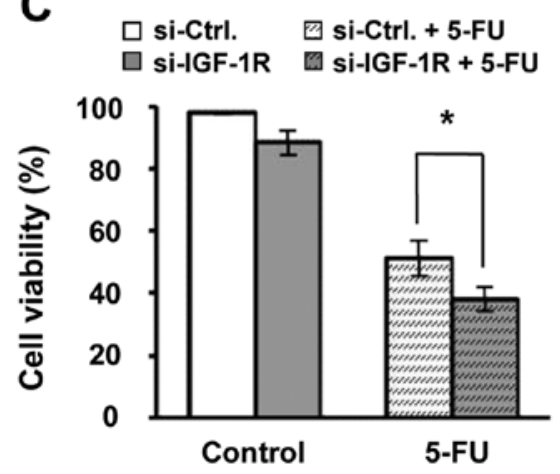

B

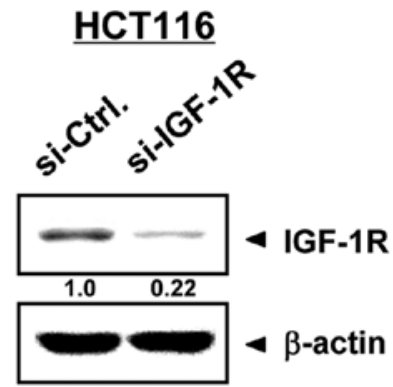

D
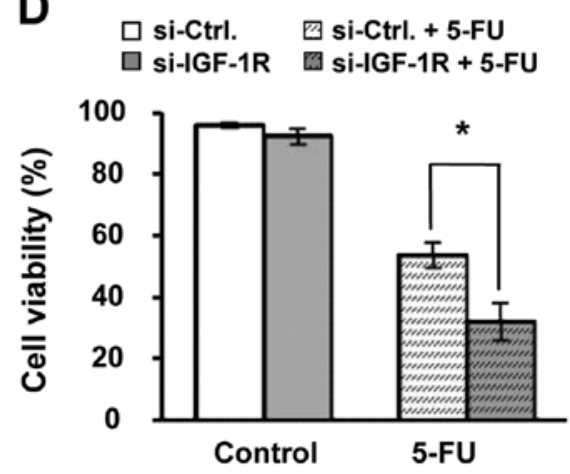

Figure 5. Repression of IGF-1R enhances 5-FU-induced inhibition of cell viability in HT29 and HCT116 cells. HT29 and HCT116 cells were transfected with si-IGF-1R or si-Ctrl as negative controls. Western blot analysis showed a significant decrease in IGF-1R expression by si-IGF-1R in (A) HT29 and (B) HCT116 cells. Next, cells were transfected as before; $6 \mathrm{~h}$ later, they were treated by 5-FU (HT29, $25 \mu \mathrm{M}$; HCT116, $10 \mu \mathrm{M})$ or not; and $48 \mathrm{~h}$ later, cell viability was measured with the Trypan blue dye-exclusion assay [(C) HT29; (D) HCT116]. Experiments were independently repeated three times with reproducible results. ${ }^{*} \mathrm{p}<0.05$ and ${ }^{* *} \mathrm{p}<0.01$. 
A

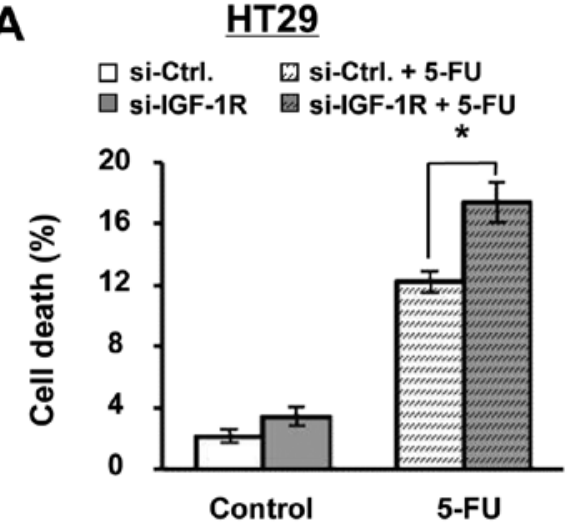
C $\square$ si-Ctrl. $\square$ si-Ctrl. + 5-FU $\square$ si-IGF-1R 싱-IGF-1R + 5-FU

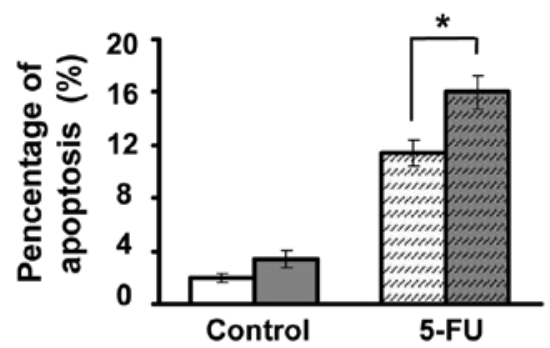

E

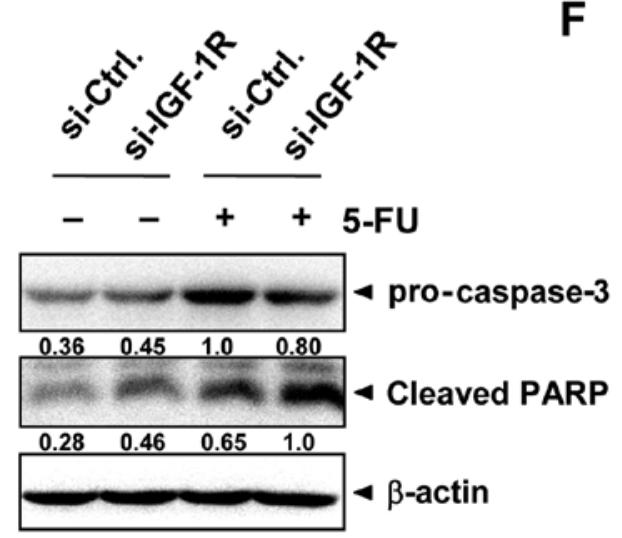

D

$\mathbf{F}$
B $\quad$ HCT116

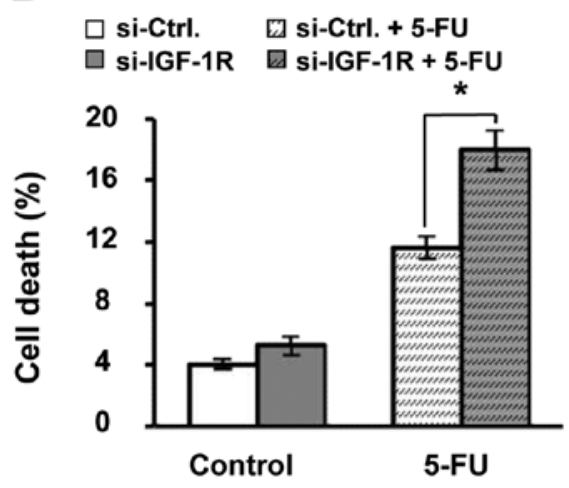

$$
\begin{array}{ll}
\hline \text { si-Ctrl. } & \text { घi-Ctrl. + 5-FU } \\
\square \text { si-IGF-1R } & \text { 卤 si-IGF-1R + 5-FU }
\end{array}
$$
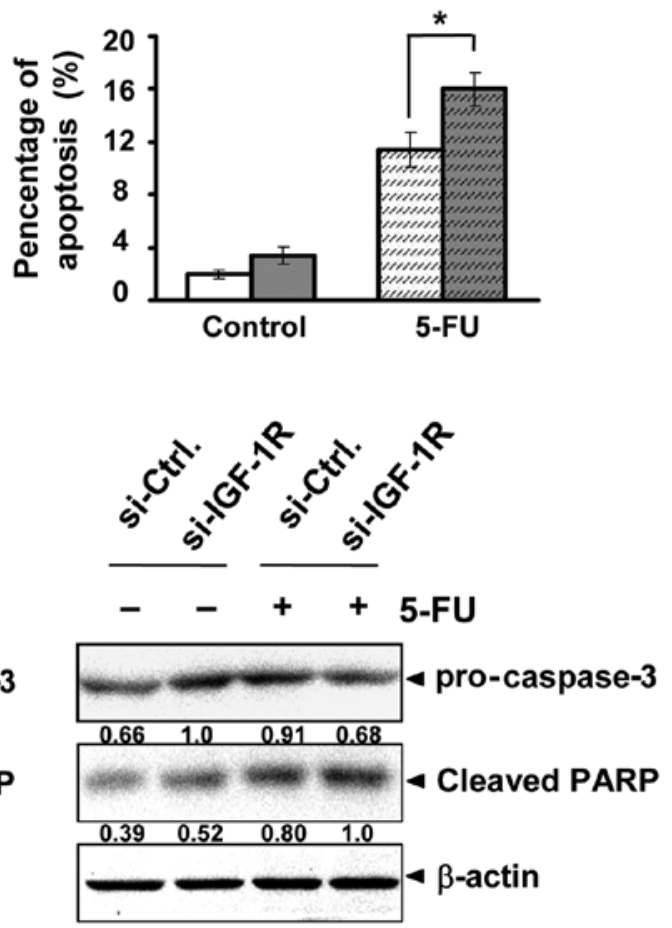

Figure 6. Repression of IGF-1R expression increases 5-FU-induced cell death. HT29 and HCT116 cells were treated as in Fig. 5. Cell death was determined by the Trypan blue dye-exclusion assay [(A) HT29; (B) HCT116]. Cell apoptosis was measured using an Annexin V-FITC apoptosis detection kit [(C) HT29; (D) HCT116]. Additionally, cell lysates were harvested, and the same protein level was loaded on SDS-PAGE gels. (E,F) The pro-caspase-3 and cleaved PARP levels were detected using western blot analysis. $\beta$-actin was used as the loading control. Data are representative of three independent experiments. "p $<0.05$.

\section{Discussion}

New therapeutic strategies and/or new adjuvant drugs are urgently needed in CRC therapy. miRNAs are suggested to be involved in cancer cell proliferation, apoptosis, migration, invasion, stemness and chemoresistance. In this study, we first revealed that miR-302a plays an important role in inhibiting IGF1-R expression and Akt signaling. This was associated with enhanced 5-FU-induced cell death and viability inhibition by miR-302a overexpression in human colon cancer cells.

Inappropriate activation of survival signaling causes uncontrolled proliferation, apoptosis resistance and increased cell motility, and it plays an important role in cancer development, progression and resistance to treatment. In CRC, one of the mechanisms causing increased activation of survival signaling, especially in the PI3K/Akt pathway, is aberrant IGF-1R expression (7). Amplified IGF-1/IGF-1R signaling is not only associated with an increased relative risk for developing $\mathrm{CRC}$, but it also contributes to CRC cell survival, invasion and metastasis (8). Activation of IGF-1R-dependent pathways has also been identified as a critical step that contributes to several mechanisms of CRC resistance to both conventional and targeted therapeutic agents (27). In addition, microRNAs were recently reported to participate in post-transcriptional regulation of IGF-1R. miR-375, miR-7 and miR-497 were reported to target IGF-1R in esophageal, tongue squamous, and colon cancer cells, respectively (28-30). In this study, miR-302a also targeted IGF-1R in human colon cancer cells.

miR-302a has been reported to inhibit prostate cancer cell proliferation by targeting Akt (19) and sensitizing testicular 
A $\quad$ HT29

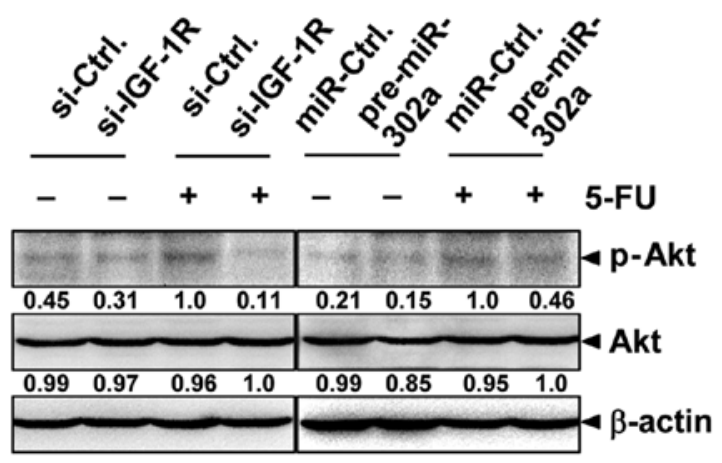

B $\quad \underline{\text { HCT116 }}$

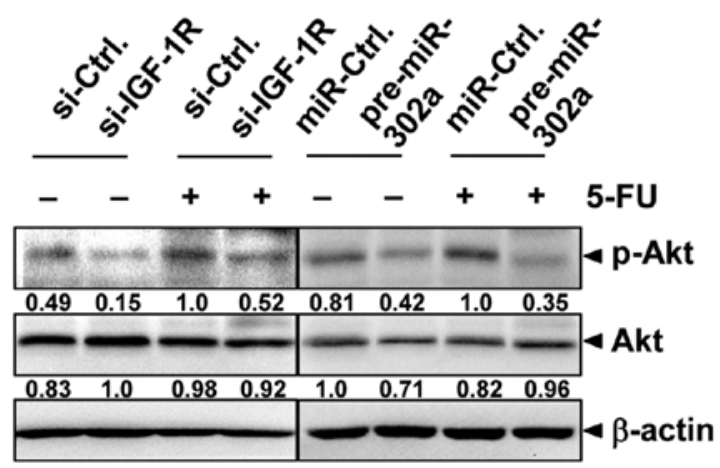

Figure 7. Overexpression of miR-302a and repression of IGF-1R produced similar changes in Akt signaling. HT29 and HCT116 cells were treated as in Figs. 2 and 5. Cell lysates were harvested, and the same protein levels were loaded onto SDS-PAGE gels. The pAkt and total Akt levels were detected using western blot analysis. $\beta$-actin was used as the loading control. Data are representative of three independent experiments.

embryonal carcinoma cells to cisplatin-induced cell death, partially through the downregulation of p21 (20). In the past, most research involving miRNA-302 has focused on its role in hESCs. Studies analyzing miR-302 function in cancer are limited. For the first time, we revealed that miR-302a could target IGF-1R and enhanced the 5-FU-induced cell death in human colon cancer cells. The targeting of miR-302a to IGF-1R was identified by a luciferase assay and the reverse expression pattern in human CRC tissues (Fig. 4). IGF-1R is a direct target of miR-302a. Through targeting IGF-1R, miR-302a inhibited downstream Akt signaling.

Akt has a major function in cell survival, proliferation, death, invasion, and migration. Constitutive activation of the PI3K/Akt pathway is a common event in many cancer types, and it is associated with a poor prognosis and reduced survival (31-33). Evidence also indicates that Akt is frequently activated, which is implicated in cancer chemoresistance $(23,26,32,34)$. Consistent with this, we found 5-FU could upregulate Akt activation; also, overexpression of miR-302a inhibited the 5-FU-induced Akt activation and enhanced the 5-FU-induced cell death in human colon cancer cells. Besides IGF-1R, miR-302a may target other genes in the PI3K/Akt pathway as reported in previous studies (17-20) and the bioinformatics analysis (Fig. 1). miR-302a is a potentially important tumor suppressor that merits further, extensive study. In addition, in our experiment, the effect of only miR-302a overexpression on cell viability and cell death was not significant, which was inconsistent with a previous study (19). This discrepancy could be due to the shorter period $(48 \mathrm{~h}$ ) of our system compared to that in the previous study ( $96 \mathrm{~h}$ to 7 days).

CRC treatment via targeting IGF1-R is now in development for clinical use. Our results suggest that overexpression of miR-302a may be a useful alternative strategy for inhibiting IGF1-R, inactivating Akt, and enhancing 5-FU-induced cell death and viability inhibition in human colon cancer cells. Targeting miR-302a may offer new therapeutic interventions in CRC.

\section{Acknowledgements}

This study was supported by grants from the National Natural Science Foundation of China (nos. 81172358 and 31100969) and the Fundamental Research Funds of Xi'an Jiaotong University (no. xjj2016076). We thank Professor H. Kuwano and S. Torii (Graduate School of Medicine, Gunma University, Maebashi, Japan) for their kind assistance. We also thank Lei Ni, Aiying Wang and Lin Yu for their technical support.

\section{References}

1. Torre LA, Bray F, Siegel RL, Ferlay J, Lortet-Tieulent J and Jemal A: Global cancer statistics, 2012. CA Cancer J Clin 65: 87-108, 2015.

2. Sung JJ, Lau JY, Goh KL and Leung WK; Asia Pacific Working Group on Colorectal Cancer: Increasing incidence of colorectal cancer in Asia: Implications for screening. Lancet Oncol 6: 871-876, 2005.

3. Temraz S, Mukherji D, Alameddine R and Shamseddine A: Methods of overcoming treatment resistance in colorectal cancer. Crit Rev Oncol Hematol 89: 217-230, 2014.

4. Longley DB, Harkin DP and Johnston PG: 5-fluorouracil: Mechanisms of action and clinical strategies. Nat Rev Cancer 3: 330-338, 2003

5. Scartozzi M, Maccaroni E, Giampieri R, Pistelli M, Bittoni A, Del Prete M, Berardi R and Cascinu S: 5-Fluorouracil pharmacogenomics: Still rocking after all these years? Pharmacogenomics 12: 251-265, 2011.

6. Vigneri PG, Tirrò E, Pennisi MS, Massimino M, Stella S, Romano C and Manzella L: The insulin/IGF system in colorectal cancer development and resistance to therapy. Front Oncol 5: 230, 2015.

7. Weber MM, Fottner C, Liu SB, Jung MC, Engelhardt D and Baretton GB: Overexpression of the insulin-like growth factor I receptor in human colon carcinomas. Cancer 95: 2086-2095, 2002.

8. Sekharam M, Zhao H, Sun M, Fang Q, Zhang Q, Yuan Z, Dan HC, Boulware D, Cheng JQ and Coppola D: Insulin-like growth factor 1 receptor enhances invasion and induces resistance to apoptosis of colon cancer cells through the Akt/Bcl-x(L) pathway. Cancer Res 63: 7708-7716, 2003.

9. Alberobello AT, D'Esposito V, Marasco D, Doti N, Ruvo M, Bianco R, Tortora G, Esposito I, Fiory F, Miele C, et al: Selective disruption of insulin-like growth factor-1 (IGF-1) signaling via phosphoinositide-dependent kinase-1 prevents the protective effect of IGF-1 on human cancer cell death. J Biol Chem 285: 6563-6572, 2010.

10. Wu WK, Law PT, Lee CW, Cho CH, Fan D, Wu K, Yu J and Sung JJ: MicroRNA in colorectal cancer: From benchtop to bedside. Carcinogenesis 32: 247-253, 2011.

11. Amirkhah R, Farazmand A, Irfan-Maqsood M, Wolkenhauer O and Schmitz U: The role of microRNAs in the resistance to colorectal cancer treatments. Cell Mol Biol (Noisy-le-grand) 61: $17-23,2015$.

12. Karaayvaz M, Zhai H and Ju J: miR-129 promotes apoptosis and enhances chemosensitivity to 5-fluorouracil in colorectal cancer. Cell Death Dis 4: e659, 2013. 
13. Zhang Y, Zheng L, Huang J, Gao F, Lin X, He L, Li D, Li Z Ding Y and Chen L: MiR-124 radiosensitizes human colorectal cancer cells by targeting PRRX1. PLoS One 9: e93917, 2014.

14. Zhou Y, Wan G, Spizzo R, Ivan C, Mathur R, Hu X, Ye X, Lu J, Fan F, Xia L, et al: miR-203 induces oxaliplatin resistance in colorectal cancer cells by negatively regulating ATM kinase. Mol Oncol 8: 83-92, 2014.

15. Suto T, Yokobori T, Yajima R, Morita H, Fujii T, Yamaguchi S, Altan B, Tsutsumi S, Asao T and Kuwano H: MicroRNA-7 expression in colorectal cancer is associated with poor prognosis and regulates cetuximab sensitivity via EGFR regulation. Carcinogenesis 36: 338-345, 2015.

16. Suh MR, Lee Y, Kim JY, Kim SK, Moon SH, Lee JY, Cha KY, Chung HM, Yoon HS, Moon SY, et al: Human embryonic stem cells express a unique set of microRNAs. Dev Biol 270: 488-498, 2004.

17. Wang Y, Baskerville S, Shenoy A, Babiarz JE, Baehner L and Blelloch R: Embryonic stem cell-specific microRNAs regulate the G1-S transition and promote rapid proliferation. Nat Genet 40 : 1478-1483, 2008.

18. Lin SL, Chang DC, Ying SY, Leu D and Wu DT: MicroRNA miR-302 inhibits the tumorigenecity of human pluripotent stem cells by coordinate suppression of the CDK 2 and CDK4/6 cell cycle pathways. Cancer Res 70: 9473-9482, 2010.

19. Zhang GM, Bao CY, Wan FN, Cao DL, Qin XJ, Zhang HL, Zhu Y, Dai B, Shi GH and Ye DW: MicroRNA-302a suppresses tumor cell proliferation by inhibiting AKT in prostate cancer. PLoS One 10: e0124410, 2015.

20. Liu L, Lian J, Zhang H, Tian H, Liang M, Yin M and Sun F: MicroRNA-302a sensitizes testicular embryonal carcinoma cells to cisplatin-induced cell death. J Cell Physiol 228: 2294-2304, 2013.

21. Hou N, Torii S, Saito N, Hosaka M and Takeuchi T: Reactive oxygen species-mediated pancreatic $\beta$-cell death is regulated by interactions between stress-activated protein kinases, p38 and c-Jun N-terminal kinase, and mitogen-activated protein kinase phosphatases. Endocrinology 149: 1654-1665, 2008.

22. Hou N, Han J, Li J, Liu Y, Qin Y, Ni L, Song T and Huang C: MicroRNA profiling in human colon cancer cells during 5-fluorouracil-induced autophagy. PLoS One 9: e114779, 2014.

23. Li B, Li J, Xu WW, Guan XY, Qin YR, Zhang LY, Law S, Tsao SW and Cheung AL: Suppression of esophageal tumor growth and chemoresistance by directly targeting the PI3K/AKT pathway. Oncotarget 5: 11576-11587, 2014.

24. Li J, Hou N, Faried A, Tsutsumi S, Takeuchi T and Kuwano H: Inhibition of autophagy by 3-MA enhances the effect of 5-FU-induced apoptosis in colon cancer cells. Ann Surg Oncol 16: 761-771, 2009.
25. Li J, Hou N, Faried A, Tsutsumi S and Kuwano H: Inhibition of autophagy augments 5-fluorouracil chemotherapy in human colon cancer in vitro and in vivo model. Eur J Cancer 46: 1900-1909, 2010

26. Vinod BS, Antony J, Nair HH, Puliyappadamba VT, Saikia M, Narayanan SS, Bevin A and Anto RJ: Mechanistic evaluation of the signaling events regulating curcumin-mediated chemosensitization of breast cancer cells to 5-fluorouracil. Cell Death Dis 4: e505, 2013.

27. Dallas NA, Xia L, Fan F, Gray MJ, Gaur P, van Buren G II, Samuel S, Kim MP, Lim SJ and Ellis LM: Chemoresistant colorectal cancer cells, the cancer stem cell phenotype, and increased sensitivity to insulin-like growth factor-I receptor inhibition. Cancer Res 69: 1951-1957, 2009.

28. Kong KL, Kwong DL, Chan TH, Law SY, Chen L, Li Y, Qin YR and Guan XY: MicroRNA-375 inhibits tumour growth and metastasis in oesophageal squamous cell carcinoma through repressing insulin-like growth factor 1 receptor. Gut 61: 33-42, 2012.

29. Jiang L, Liu X, Chen Z, Jin Y, Heidbreder CE, Kolokythas A, Wang A, Dai Y and Zhou X: MicroRNA-7 targets IGF1R (insulin-like growth factor 1 receptor) in tongue squamous cell carcinoma cells. Biochem J 432: 199-205, 2010.

30. Guo ST, Jiang CC, Wang GP, Li YP, Wang CY, Guo XY, Yang RH, Feng Y, Wang FH, Tseng HY, et al: MicroRNA-497 targets insulin-like growth factor 1 receptor and has a tumour suppressive role in human colorectal cancer. Oncogene 32: 1910-1920, 2013

31. Yoshioka A, Miyata H, Doki Y, Yasuda T, Yamasaki M, Motoori M, Okada K, Matsuyama J, Makari Y, Sohma I, et al: The activation of Akt during preoperative chemotherapy for esophageal cancer correlates with poor prognosis. Oncol Rep 19: 1099-1107, 2008

32. Liu P, Cheng H, Roberts TM and Zhao JJ: Targeting the phosphoinositide 3-kinase pathway in cancer. Nat Rev Drug Discov 8: 627-644, 2009 .

33. Faried LS, Faried A, Kanuma T, Aoki H, Sano T, Nakazato T, Tamura T, Kuwano $\mathrm{H}$ and Minegishi T: Expression of an activated mammalian target of rapamycin in adenocarcinoma of the cervix: A potential biomarker and molecular target therapy. Mol Carcinog 47: 446-457, 2008.

34. Faried LS, Faried A, Kanuma T, Nakazato T, Tamura T, Kuwano $\mathrm{H}$ and Minegishi $\mathrm{T}$ : Inhibition of the mammalian target of rapamycin (mTOR) by rapamycin increases chemosensitivity of CaSki cells to paclitaxel. Eur J Cancer 42: 934-947, 2006. 